\title{
ON NON-LOCAL STABILITY PROPERTIES OF EXTREMUM SEEKING CONTROL
}

\author{
Y. Tan, D. Nešić and I.M.Y.Mareels ${ }^{*, 1}$ \\ * The Department of Electrical $\&$ Electronics, \\ The University of Melbourne, Parkview, VIC 3010
}

\begin{abstract}
We revisit the extremum seeking scheme whose local stability properties were analyzed in (Krstić and Wang, 2000) and propose its simplified version that still achieves extremum seeking. We show under slightly stronger conditions that this simplified scheme achieves extremum seeking from arbitrarily large domain of initial conditions if the parameters in the controller are appropriately adjusted. This non-local convergence result is proved by showing semi-global practical stability of the closed-loop system with respect to the design parameters. Moreover, we show at the same time that reducing the parameters typically slows down the convergence of the extremum seeking controller. Hence, the control designer faces a tradeoff between the size of the domain of attraction and the speed of convergence when tuning the extremum seeking controller. We present a simulation example to illustrate our results.
\end{abstract}

Keywords: Nonlinear, Extremum seeking, Averaging, Singular perturbations, Stability.

\section{INTRODUCTION}

In many control applications the reference-tooutput map has an extremum and the control objective is to regulate the output close to this extremum. For instance, consider the system:

$$
\dot{\mathbf{x}}=f(\mathbf{x}, u) ; \quad y=h(\mathbf{x})
$$

and suppose that there exists an $\mathbf{x}^{*}$ such that $y^{*}=h\left(\mathbf{x}^{*}\right)$ is an extremum of the mapping $h(\cdot)$. Due to uncertainty, it is often reasonable to assume that neither $\mathbf{x}^{*}$ nor $h(\cdot)$ are known to the control designer. The main objective in extremum seeking control is to force the solutions of the closed loop system to eventually converge to $\mathbf{x}^{*}$ and to do so without precise knowledge about $\mathbf{x}^{*}$ or $h(\cdot)$.

Extremum seeking control is an old topic that was popular in 1950's and 1960's. It focused on

\footnotetext{
1 This research was supported by the Australia Research Council under the Discovery Project DP0344784
}

finding the optimal value of a static mapping and stability issues were largely ignored (see (Krstić and Wang, 2000)). It was not until the year 2000 that stability of an extremum seeking feedback scheme was proved rigorously in (Krstić and Wang, 2000). This sparked a new interest in the area and generated numerous new results and applications (M. Guay, 2004; Krstić, 2000; Peterson and Stefanopoulou, 2004; Popović et al., 2003; H.-H. Wang and Krstić, 2000; Wang and Krstić, 2000).

The analysis in (Krstić and Wang, 2000) was based on classical singular perturbations and averaging results and only a local stability result was proved. In other words, results in (Krstić and Wang, 2000) do not say anything about the actual domain of attraction. In most engineering applications it is very useful to obtain estimates of the domain of attraction.

The main purpose of this paper is to characterize non-local stability properties of an extremum seek- 
ing controller. In other words, our results include explicit statements about the achieved domain of attraction for the closed loop. We show under appropriate conditions that extremum seeking controller achieves semi-global practical stability of the closed loop system. In other words, given an arbitrarily large set of initial conditions $B_{\Delta}$ and an arbitrarily small neighborhood $B_{\delta}$ of the state $\mathbf{x}^{*}$ where the output achieves its extremum $y^{*}=h\left(\mathbf{x}^{*}\right)$, it is possible to adjust the controller parameters so that all solutions starting from the set $B_{\Delta}$ eventually converge to $B_{\delta}$. At the same time we show that reducing the parameters in the controller typically reduces the speed of convergence of the algorithm and this poses a tradeoff that the controller designer needs to resolve when tuning the controller. Note that this is a much stronger statement than the local result proved in (Krstić and Wang, 2000). To the best of our knowledge, this is the first proof of non-local and semi-global practical stability properties of extremum seeking controllers with explicit bounds of convergence speed.

Finally, we emphasize that our proof technique is novel and is based on Lyapunov techniques and recent new developments in the theory of averaging (Nešić and Teel, 2001; Teel, 2000; A. R. Teel and Aeyels, 1999) and singular perturbations (Christofides and Teel, 1996; Teel et al., 2003) that are tailored for analysis of semi-global practical stability of systems that exhibit time scale separation. Similar to (Krstić and Wang, 2000) we show that the closed loop system with an extremum seeking controller exhibits two time scales. We consider a simplified extremum seeking controller from (Krstić and Wang, 2000). Then, we show how to tune the controller parameters to achieve semi-global practical stability of the closed loop. An example is included to illustrate the design trade-off issues.

\section{PRELIMINARIES}

The following definition and lemmas are need in the sequel.

Definition 1. Consider the parameterized family of systems:

$$
\dot{\mathbf{x}}=\mathbf{f}\left(t, \mathbf{x}, \epsilon_{1}, \epsilon_{2}, \ldots, \epsilon_{\ell}\right),
$$

where $\mathbf{x} \in R^{n}$ and $\epsilon_{i} \in R_{>0}$ for all $i=1,2, \ldots, \ell$. The system (1) is said to be semi-globally practically asymptotically (SPA) stable in $\left[\epsilon_{1}, \epsilon_{2}, \cdots, \epsilon_{\ell}\right]$, if there exists $\beta \in \mathcal{K} \mathcal{L}$ (Khalil, 2002) such that the following holds: for each pair of strictly positive real numbers $(\Delta, \nu)$, there exists $\epsilon_{1}^{*}>0$ and for any $\epsilon_{1} \in$ $\left(0, \epsilon_{1}^{*}\right)$ there exists $\epsilon_{2}^{*}=\epsilon_{2}^{*}\left(\epsilon_{1}\right)>0$ and for any $\epsilon_{2} \in$ $\left(0, \epsilon_{2}^{*}\right)$ there exists $\epsilon_{3}^{*}=\epsilon_{3}^{*}\left(\epsilon_{1}, \epsilon_{2}\right)>0$ and for any $\epsilon_{3} \in\left(0, \epsilon_{3}^{*}\right) \cdots$, there exists $\epsilon_{\ell}^{*}\left(\epsilon_{1}, \epsilon_{2}, \ldots, \epsilon_{\ell-1}\right)>0$ such that for any $\epsilon_{\ell} \in\left(0, \epsilon_{\ell}^{*}\right)$, the solutions of (1) with the parameters $\left[\epsilon_{1}, \epsilon_{2}, \ldots, \epsilon_{\ell}\right]$ satisfy:

$$
|\mathbf{x}(t)| \leq \beta\left(\left|\mathbf{x}_{0}\right|,\left(\epsilon_{1} \cdot \epsilon_{2} \cdots \epsilon_{\ell}\right)\left(t-t_{0}\right)\right)+\nu,
$$

for all $t \geq t_{0} \geq 0, \mathbf{x}\left(t_{0}\right)=\mathbf{x}_{0}$ with $\left|\mathbf{x}_{0}\right| \leq \Delta$. Moreover, if under the stated conditions we have that:

$$
|\mathbf{x}(t)| \leq \beta\left(\left|\mathbf{x}_{0}\right|,\left(\epsilon_{1} \cdot \epsilon_{2} \cdots \epsilon_{\ell-1}\right)\left(t-t_{0}\right)\right)+\nu,
$$

$\forall t \geq t_{0} \geq 0$, then we say that the system (1) is SPA stable in $\left[\epsilon_{1}, \epsilon_{2}, \ldots, \epsilon_{\ell}\right]$, uniformly in $\epsilon_{\ell}$.

Remark 1: Definition 1 is not the standard definition of semi-global practical stability. First, the definition is stated for systems with multiple parameters $\epsilon_{1} \cdot \epsilon_{2} \cdots \epsilon_{\ell}$. The order of the parameters $\left[\epsilon_{1}, \epsilon_{2}, \ldots, \epsilon_{\ell}\right]$ in Definition 1 is very important. Indeed, first we find $\epsilon_{1}^{*}$ and then fix $\epsilon_{1} \in\left(0, \epsilon_{1}^{*}\right)$. Then, we find $\epsilon_{2}^{*}=\epsilon_{2}^{*}\left(\epsilon_{1}\right)$ and fix $\epsilon_{2} \in\left(0, \epsilon_{2}^{*}\right)$, and so on. Finally, with the choice of parameters $\left[\epsilon_{1}, \ldots, \epsilon_{\ell}\right]$ the trajectories of the system (1) should satisfy the stability bound (2). Second, note that the convergence speed in Definition 1 depends on the parameters, which is different from standard definitions of SPA stability. However, the overshoot are uniform in parameters $\left(\beta\right.$ is independent of $\epsilon_{i}$ )

The proof of the following fact follows similar steps as that of (Nešić and Teel, 2001, Corollary 1)

Lemma 1. Consider the parameterized family of nonlinear systems

$$
\dot{\mathbf{x}}=\mathbf{g}\left(\frac{t}{\epsilon}, \mathbf{x}, a\right),
$$

where $\mathbf{x} \in R^{n}, \epsilon, a>0$. The function $\mathbf{g}(t, \mathbf{x}, a)$ is locally Lipschitz in $\mathbf{x}$ and $a$, uniformly in $t$, as well as periodic in $t$ of period $2 \pi$. Define $\mathbf{g}_{a v}(\mathbf{x}, a) \triangleq$ $\frac{1}{2 \pi} \int_{0}^{2 \pi} \mathbf{g}(\tau, \mathbf{x}, a) d \tau$. Suppose that the average system $\dot{\mathbf{y}}=\mathbf{g}_{a v}(\mathbf{y}, a)$ is SPA stable in $a$. Then, the actual system (4) is SPA stable in $[a, \epsilon]$, uniformly in $\epsilon$.

In order to state the next lemma, we consider the following nonlinear system

$$
\begin{aligned}
\frac{d \mathbf{x}}{d t} & =\mathbf{f}\left(t, \mathbf{x}, \mathbf{z}, \epsilon_{1}, \epsilon_{2}, \cdots, \epsilon_{\ell}\right) \\
\epsilon_{\ell} \frac{d \mathbf{z}}{d t} & =\mathbf{g}\left(t, \mathbf{x}, \mathbf{z}, \epsilon_{\ell}\right),
\end{aligned}
$$

where $\mathbf{x} \in R^{n}, \mathbf{z} \in R^{q}$ and $\left[\epsilon_{1}, \epsilon_{2}, \cdots, \epsilon_{\ell-1}\right] \in$ $R^{\ell-1}$. Let $\epsilon_{\ell}=0$, the state vector $\mathbf{z}$ becomes instantaneous and (5) takes the form

$$
\begin{aligned}
\frac{d \mathbf{x}}{d t} & =\mathbf{f}\left(t, \mathbf{x}, \mathbf{z}_{s}, \epsilon_{1}, \epsilon_{2}, \cdots, \epsilon_{\ell-1}, 0\right) \\
0 & =\mathbf{g}\left(t, \mathbf{x}, \mathbf{z}_{s}, 0\right),
\end{aligned}
$$

where $\mathbf{z}_{s}$ denotes a quasi-steady state for the fast state vector $\mathbf{z}$. With $\mathbf{z}_{s}=\mathbf{h}(t, \mathbf{x})$, the following reduced system is obtained

$$
\frac{d \mathbf{x}}{d t}=\mathbf{f}\left(t, \mathbf{x}, \mathbf{h}(t, \mathbf{x}), \epsilon_{1}, \epsilon_{2}, \cdots, \epsilon_{\ell-1}, 0\right) .
$$


Introducing $\mathbf{y}=\mathbf{z}-\mathbf{h}(t, \mathbf{x}), \tau=\frac{t-t_{0}}{\epsilon_{\ell}}$ and setting $\epsilon_{\ell}=0$, the boundary layer satisfies

$$
\frac{d \mathbf{y}}{d \tau}=\mathbf{g}\left(t_{0}, \mathbf{x}, \mathbf{h}\left(t_{0}, \mathbf{x}\right)+\mathbf{y}, 0\right) .
$$

The proof of the following lemma follows almost the same steps as the proof of the main result in (Teel et al., 2003).

Lemma 2. Suppose the following conditions hold:

(1) The algebraic equation $\mathbf{g}\left(t, \mathbf{x}, \mathbf{z}_{s}, 0\right)=0$ possesses a unique root $\mathbf{z}_{s}=\mathbf{h}(t, \mathbf{x})$, where $\mathbf{h}$ : $R^{n} \rightarrow R^{q}$ and its partial derivatives $\frac{\partial \mathbf{h}}{\partial \mathbf{x}}$ are locally Lipschitz, uniformly in $t$.

(2) The reduced system (7) is SPA in $\left[\epsilon_{1}, \epsilon_{2}, \cdots, \epsilon_{\ell-1}\right]$.

(3) The equilibrium $\mathbf{y}=\mathbf{0}$ of the boundary layer system in (8) is globally asymptotically stable, uniformly in $\mathbf{x}$ and $t_{0}$.

Then, the system (5) is SPA stable in $\left[\epsilon_{1}, \epsilon_{2}, \cdots, \epsilon_{\ell}\right]$, uniformly in $\epsilon_{\ell}$ (with the time scale $t$ ).

We show in the next section that our extremum seeking scheme exhibits several time scales that will be characterized by several different parameters and the closed loop system has the form of (1). We will show that the closed loop system of the extremum seeking is SPA stable in the sense of Definition 1.

\section{PROBLEM FORMULATION}

In this paper, our attention is centered on the following SISO nonlinear model

$$
\begin{aligned}
\dot{\mathbf{x}} & =\mathbf{f}(\mathbf{x}, u) \\
y & =h(\mathbf{x}),
\end{aligned}
$$

where $^{2} \mathbf{f}: R^{n} \times R \rightarrow R^{n}$ and $h: R^{n} \rightarrow R$. Consider a family of control laws: $u=\alpha(\mathbf{x}, \theta)$, where $\theta \in R$ is a scalar parameter. The closed-loop system is then

$$
\dot{\mathbf{x}}=\mathbf{f}(\mathbf{x}, \alpha(\mathbf{x}, \theta)),
$$

whose equilibrium is parameterized by $\theta$. For simplicity, we assume that $\theta, u$ and $y$ are scalars and the control law in (10) is static. All of these assumptions can be removed but they will simplify the presentation. The following assumption is the same as (Krstić and Wang, 2000, Assumption 2.1).

Assumption 1: There exists a function $1: R \rightarrow R^{n}$ such that

$$
f(\mathbf{x}, \alpha(\mathbf{x}, \theta))=0, \quad \text { iff } \quad \mathbf{x}=\mathbf{l}(\theta) .
$$

Assumption 2 is a natural extension of (Krstić and Wang, 2000, Assumption 2.2) that is needed to

2 In the sequel, all functions are assumed to be sufficiently smooth (all derivatives that we need are continuous). prove non-local stability properties of the closedloop.

Assumption 2: For each $\theta \in R$, the equilibrium $\mathbf{x}=\mathbf{l}(\theta)$ of the system (10) is globally asymptotically stable, uniformly in $\theta$.

Assumption 3: Denoting $Q(\cdot)=h \circ \mathbf{l}(\cdot)$, there exists a global maximum $\theta^{*}$ of $Q(\cdot)$ and, moreover, the following holds ${ }^{3}$ :

$$
\frac{\partial Q}{\partial \theta}(\theta)=0 \quad \text { iff } \quad \theta=\theta^{*} ; \quad \frac{\partial^{2} Q}{\partial \theta^{2}}\left(\theta^{*}\right)<0 .
$$

Remark 2 Assumption 3 is a stronger version of (Krstić and Wang, 2000, Assumption 2.3), where it was assumed that

$$
\frac{\partial Q}{\partial \theta}\left(\theta^{*}\right)=0 ; \quad \frac{\partial^{2} Q}{\partial \theta^{2}}\left(\theta^{*}\right)<0 .
$$

Using conditions (13), only local stability properties of the extremum seeking scheme were analyzed in (Krstić and Wang, 2000). We use the stronger condition (12) in Assumption 2 to show a stronger (non-local) stability property of the extremum seeking scheme.

\section{MAIN RESULTS}

Our main result is stated and proved in this section. We simplify the extremum seeking controller from (Krstić and Wang, 2000) and prove that this simplified scheme still achieves extremum seeking. We use the same method for tuning the controller parameters as in (Krstić and Wang, 2000). More importantly, under the above assumptions, we prove a non-local stability result extending the main result in (Krstić and Wang, 2000). Our assumptions are natural when investigating non-local results.

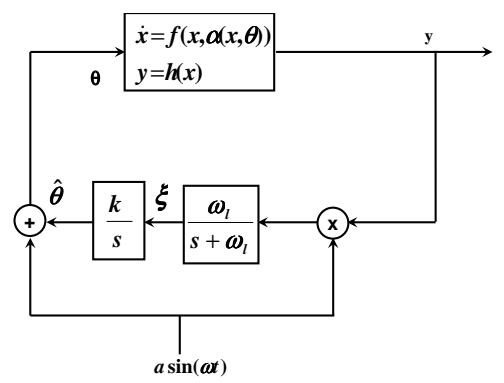

Fig. 1. A extremum seeking feedback scheme

Consider the extremum seeking controller given in Figure 1. Note that this scheme is a simplified version of the scheme considered in (Krstić and Wang, 2000), where an extra linear filter was used to filter the output $y$ of the plant. The following equations describe the closed loop system:

3 Without loss of generality we assume that the extremum is a maximum. 


$$
\begin{aligned}
& \dot{\mathbf{x}}=\mathbf{f}(\mathbf{x}, \alpha(\mathbf{x}, \hat{\theta}+a \cdot \sin (\omega \cdot t)) \\
& \dot{\hat{\theta}}=k \cdot \xi \\
& \dot{\xi}=-\omega_{l} \cdot \xi+\omega_{l} \cdot y \cdot a \cdot \sin (\omega \cdot t) .
\end{aligned}
$$

Introduce the change of the coordinates, $\tilde{\mathbf{x}}=\mathbf{x}-$ $\mathbf{x}^{*}, \tilde{\theta}=\hat{\theta}-\theta^{*}, \tilde{\xi}=\xi$ and note that the point $\left(\mathbf{x}^{*}, \theta^{*}, 0\right)$ is not an equilibrium point of the system (14) whenever $a \neq 0$. Nevertheless, we will show that the system in new coordinates is SPA stable, which would ensure extremum seeking. The system in new coordinates takes the following form:

$$
\begin{aligned}
& \dot{\tilde{\mathbf{x}}}=\mathbf{f}\left(\tilde{\mathbf{x}}+\mathbf{x}^{*}, \alpha\left(\tilde{\mathbf{x}}+\mathbf{x}^{*}, \tilde{\theta}+\theta^{*}+a \cdot \sin (\omega \cdot t)\right)\right. \\
& \dot{\tilde{\theta}}=k \cdot \tilde{\xi} \\
& \dot{\tilde{\xi}}=-\omega_{l} \cdot\left[\tilde{\xi}-h\left(\tilde{\mathbf{x}}+\mathbf{x}^{*}\right) \cdot a \cdot \sin (\omega \cdot t)\right] .
\end{aligned}
$$

Fix $\omega_{L}, K \in R_{>0}$ and define

$$
\omega_{l} \triangleq \omega \delta \omega_{L} ; \quad k \triangleq \omega \delta K
$$

where $\delta$ is a new "small" parameter. We introduce the new time " $\tau=\omega \cdot t$ " and obtain:

$$
\begin{aligned}
\omega \frac{d \tilde{\mathbf{x}}}{d \tau} & =\mathbf{f}\left(\tilde{\mathbf{x}}+\mathbf{x}^{*}, \alpha\left(\tilde{\mathbf{x}}+\mathbf{x}^{*}, \tilde{\theta}+\theta^{*}+a \cdot \sin (\tau)\right)\right. \\
\frac{d \tilde{\theta}}{d \tau} & =\delta \cdot K \cdot \tilde{\xi} \\
\frac{d \tilde{\xi}}{d \tau} & =-\delta \cdot \omega_{L} \cdot\left[\tilde{\xi}-h\left(\tilde{\mathbf{x}}+\mathbf{x}^{*}\right) \cdot a \cdot \sin (\tau)\right]
\end{aligned}
$$

This system exhibits the same two time-scale structure as in (Krstić and Wang, 2000). Our first main result is stated next:

Theorem 1. Under Assumptions 1-3, the closedloop system (15) is SPA stable in $\left[a^{2}, \delta, \omega\right]$.

A more complicated extremum seeking scheme was investigated in (Krstić and Wang, 2000):

$$
\begin{aligned}
& \dot{\mathbf{x}}=\mathbf{f}(\mathbf{x}, \alpha(\mathbf{x}, \hat{\theta}+a \cdot \sin (\omega \cdot t)) \\
& \dot{\hat{\theta}}=k \cdot \xi \\
& \dot{\xi}=-\omega_{l} \cdot \xi+\omega_{l} \cdot(y \cdot-\eta) a \cdot \sin (\omega \cdot t) \\
& \dot{\eta}=-\omega_{h} \cdot \eta+\omega_{h} \cdot y,
\end{aligned}
$$

where besides (16), we also use $\omega_{h} \triangleq \omega \delta \omega_{H}$ for some fixed $\omega_{H}>0$. The proof of Theorem 1 can be modified in a straightforward manner to prove the following result, whose proof is omitted for space reasons.

Theorem 2. Under Assumptions 1-3, the closedloop system (18) is SPA stable in $\left[a^{2}, \delta, \omega\right]$.

Remark 3: Theorems 1 and 2 are stronger results than (Krstić and Wang, 2000, Theorem 1) since we prove SPA stability, as opposed to local stability in
(Krstić and Wang, 2000). However, our results are stated under stronger assumptions (Assumptions 1 - 3) than those in (Krstić and Wang, 2000). Assumptions 1 - 3 appear to be natural when nonlocal stability is investigated. Moreover, we note that it is not crucial in Assumptions $1-3$ that all conditions hold globally. For instance, the global maximum assumption can be sometimes replaced by the local maximum assumption:

$\frac{\partial Q}{\partial \theta}(\theta)=0$ iff $\theta \in \mathcal{D}$ and $\theta=\theta^{*} ; \quad \frac{\partial^{2} Q}{\partial \theta^{2}}\left(\theta^{*}\right)<0$,

where $\mathcal{D}$ is a neighborhood of $\theta^{*}$. We concentrate only on the case $\mathcal{D}=R$ for simplicity. We note that these conditions are not very restrictive, whereas their global version is (Assumptions 2 and 3). Similarly, we could assume only local stability in Assumption 2. If all of our assumptions were regional (as opposed to global) we could still state regional results on extremum seeking which would be still stronger than the local results in (Krstić and Wang, 2000).

Remark 4: Note that Theorem 1 specifies a rule for tuning the extremum seeking controller via Definition 1 . Indeed, given $(\Delta, \nu)$ with which we would like (2) to hold, we first fix $a^{2}$ sufficiently small, then $\delta$ sufficiently small, then $\omega$ sufficiently small and finally we calculate $\omega_{l}$ and $k$ via (16). This gives us the desired parameters for the controller that will achieve non-local extremum seeking. We note that it is possible to obtain estimates of all of these parameters from our proofs but these estimates will typically be very conservative.

Remark 5: Note that since $h(\cdot)$ is continuous then for any $\nu>0$ there exists $\nu_{1}>0$ such that

$$
|\tilde{\mathbf{x}}| \leq \delta_{1} \Longrightarrow\left|h\left(\tilde{\mathbf{x}}+\mathbf{x}^{*}\right)-y^{*}\right| \leq \nu .
$$

Theorem 1 can be interpreted as follows. For any $(\Delta, \nu)$, there exist parameters $\left[a^{2}, \delta, \omega\right]$ such that for all $|(\tilde{\mathbf{x}}, \tilde{\theta}, \tilde{\xi})| \leq \Delta$, we have that

$$
\lim \sup _{t \rightarrow \infty}\left|y(t)-y^{*}\right| \leq \nu .
$$

In other words, the output of the system can be regulated arbitrarily close to the extremum value $y^{*}$ from arbitrarily large set of initial conditions by adjusting the parameters $\left[a^{2}, \delta, \omega\right]$ in the controller. In particular, the parameters $\left[a^{2}, \delta, \omega\right]$ are chosen so that Definition 1 holds with $\left(\Delta, \nu_{1}\right)$ and $\nu_{1}$ is defined in (19).

Remark 6: Note that $a, \delta$ and $\omega$ in extremum seeking design are usually small when given a larger domain of attraction, there is an obvious tradeoff in the design of an extremum seeking controller. Indeed, while the domain of attraction will be increased and we would converge closer to the extremum if we reduce the parameters $\left[a^{2}, \delta, \omega\right]$, this would typically slow down the convergence speed of the scheme. It is an interesting research topic to obtain extremum seeking schemes whose speed 
of convergence does not change while the domain of attraction is enlarged by tuning the controller parameters.

Remark 7: In the convergence speed analysis of the extremum seeking scheme, the "worst case" convergence speed is considered. That is, the convergence speed of the overall system depends on the convergence speed of the slowest sub-system in order to ensure a uniform bound of the overall system.

Remark 8: There are three parameters $(a, \delta, \omega)$ that the designer can tune in the extremum controller from (17). All other controller parameters are determined via (16). We note that there is a slight difference between the way that we tune the parameters and the way this was done in (Krstić and Wang, 2000). Indeed, (Krstić and Wang, 2000, Theorem 1) states that under appropriate assumptions the system (17) is locally stable in $\left[a^{2},\left(\begin{array}{l}\delta \\ \omega\end{array}\right)\right]$, where $\left(\begin{array}{c}\delta \\ \omega\end{array}\right)$ is treated as a parameter vector. However, a closer inspection of (Krstić and Wang, 2000) shows that (Krstić and Wang, 2000, Theorem 1) can be restated so that under the same assumptions the system (17) is locally stable in $[a, \delta, \omega]$. Hence, we do not make a distinction between the controller tuning scheme in (Krstić and Wang, 2000, Theorem 1) and our Theorem 1.

Sketch of Proof of Theorem 1: The system (17) has 2 time scales: fast dynamics $\tilde{\mathbf{x}}$ and slow dynamics $(\tilde{\theta}, \tilde{\xi})$ when $\omega$ is a small positive constant. We next use the singular perturbation method. To this end, we set $\omega=0$ and "freeze" $\tilde{\mathbf{x}}$ at its "equilibrium", $\tilde{\mathbf{x}}=\mathbf{l}\left(\theta^{*}+\tilde{\theta}+a \cdot \sin (\tau)\right)-\mathbf{x}^{*}$ to obtain the reduced system in variables $\left(\xi_{r}, \theta_{r}\right)$ :

$$
\left[\begin{array}{c}
\frac{d \theta_{r}}{d \tau} \\
\frac{d \xi_{r}}{d \tau}
\end{array}\right]=\delta\left[\begin{array}{c}
K \cdot \xi_{r} \\
-\omega_{L}\left[\xi_{r}-P\left(\theta^{*}, \theta_{r}, a \cdot \sin (\tau)\right)\right]
\end{array}\right]
$$

where $P\left(\theta^{*}, \theta_{r}, a \cdot \sin (\tau)\right) \triangleq Q\left(\theta^{*}+\theta_{r}+a \cdot \sin (\tau)\right)$. $a \cdot \sin (\tau)$. It is more convenient to write in the following time scale $s:=\delta \tau$ :

$$
\left[\begin{array}{c}
\frac{d \theta_{r}}{d s_{r}} \\
\frac{d \xi_{r}}{d s}
\end{array}\right]=\left[\begin{array}{c}
K \cdot \xi_{r} \\
-\omega_{L}\left[\xi_{r}-P\left(\theta^{*}, \theta_{r}, a \cdot \sin \left(\frac{s}{\delta}\right)\right)\right]
\end{array}\right]
$$

We introduce an auxiliary average system for (21):

$$
\left[\begin{array}{l}
\frac{d \zeta}{d s} \\
\frac{d \varrho}{d s}
\end{array}\right]=\left[\begin{array}{c}
K \varrho \\
-\omega_{L} \varrho+\frac{\omega_{L}}{2 \pi} \int_{0}^{2 \pi} P\left(\theta^{*}, \theta_{r}, a \cdot \sin (s)\right) d s
\end{array}\right]
$$

By Taylor expansions (Edwards and Penney, 1998), there exists a continuous function $g$ such that:

$$
\begin{aligned}
& \frac{1}{2 \pi} \int_{0}^{2 \pi} Q\left(\theta^{*}+\theta_{r}+a \cdot \sin (s)\right) \cdot a \cdot \sin (s) d s \\
= & \frac{a^{2}}{2} \cdot \frac{\partial Q}{\partial \zeta}\left(\zeta+\theta^{*}\right)+\frac{a^{4}}{2} g\left(\zeta, \theta^{*}, a\right) .
\end{aligned}
$$

where

$$
g\left(\zeta+\theta^{*}, a\right) \triangleq \frac{1}{2 \pi} \int_{0}^{2 \pi}[\sin (\nu)]^{4} d \nu \cdot r\left(a, \theta^{*}, \zeta\right)
$$

and $r\left(a, \theta^{*}, \zeta\right) \triangleq \int_{0}^{1}(1-\eta)^{2} \cdot \frac{\partial^{3} Q}{\partial \zeta^{3}}\left(\zeta+\theta^{*}+\right.$ $\eta a \sin (s)) d \eta$. Therefore, (22) can be re-written as follows,

$$
\begin{aligned}
& \frac{d \mathbf{z}}{d s}=\left[\begin{array}{c}
K \varrho \\
-\omega_{L} \varrho+\omega_{L}\left[\frac{a^{2}}{2} \frac{\partial Q}{\partial \zeta}\left(\zeta+\theta^{*}\right)+\frac{a^{4}}{2} g\left(\zeta, \theta^{*}, a\right)\right]
\end{array}\right] \\
& \triangleq f_{a v}\left(a, \theta^{*}, \mathbf{z}\right),
\end{aligned}
$$

where we used $\mathbf{z} \triangleq[\zeta, \varrho]^{T}$. The proof of Theorem 1 is carried out in three steps:

Step 1: We show that the average system (24) is SPA stable in $a^{2}$ (with time " $s$ "). We constructe a positive definite and radially unbounded Lyapunov function $V(\zeta, \varrho)=\frac{1}{2} \zeta^{2}+\frac{1}{c} \zeta \cdot \varrho+\left(\frac{1}{2}+\frac{1}{2 c^{2}}\right) \varrho^{2}$, where $c \triangleq \frac{K}{\omega_{L}}$. Taking derivative of $V$ along solutions of (24), we can show that system (24) is SPA stable in $a^{2}$, where the underlying time scale is $s$.

Step 2: By using Lemma 1, the system (21) is SPA stable in $\left[a^{2}, \delta\right]$, uniformly in $\delta$ (with the time scale $s)$. Using $s=\delta \tau$ this immediately implies that the system $(20)$ is SPA stable in $\left[a^{2}, \delta\right]$ (with the time scale $\tau)$.

Step 3: According to Assumption 2, the boundary layer system,

$$
\begin{aligned}
\frac{d \overline{\mathbf{x}}}{d t}= & \mathbf{f}\left(\overline{\mathbf{x}}+\mathbf{l}\left(\omega t_{0}\right), \alpha\left(\overline{\mathbf{x}}+\mathbf{l}\left(\omega t_{0}\right), \theta\left(\omega t_{0}\right)\right)\right. \\
& +\omega \cdot \frac{\partial \mathbf{l}}{\partial \tilde{\theta}} \cdot \delta \cdot K \xi \\
= & \mathbf{f}\left(\overline{\mathbf{x}}+\mathbf{l}(0), \alpha\left(\overline{\mathbf{x}}+\mathbf{l}(0), \theta^{*}+\tilde{\theta}(0)\right)\right),
\end{aligned}
$$

is asymptotically stable. In Step 2 we showed that the reduced system of the singularly perturbed system (17) is SPA stable in $\left[a^{2}, \delta\right]$. Hence, using Lemma 2, the system (17) is SPA stable in $\left[a^{2}, \delta, \omega\right]$, uniformly in $\omega$ (with the time scale $\tau$ ). Noting that $\tau=\omega t$, this implies directly that the system (15) is SPA stable in $\left[a^{2}, \delta, \omega\right]$ (with the time scale $\mathrm{t}$ ), which completes the proof.

\section{AN ILLUSTRATIVE EXAMPLE}

The purpose of this example is to illustrate SPA stability: a larger domain of attraction leads to a slower convergence speed as parameters are reduced. Consider the nonlinear system:

$$
\dot{x}=-x+u^{2}+4 u ; \quad y=-(x+4)^{2}
$$

It is obvious that when $x=-4, y$ reaches its global maximum $y^{*}=0$. Let control input $u=\theta$, we have $\theta^{*}=-2, x^{*}=-4$ and $y^{*}=0$. 

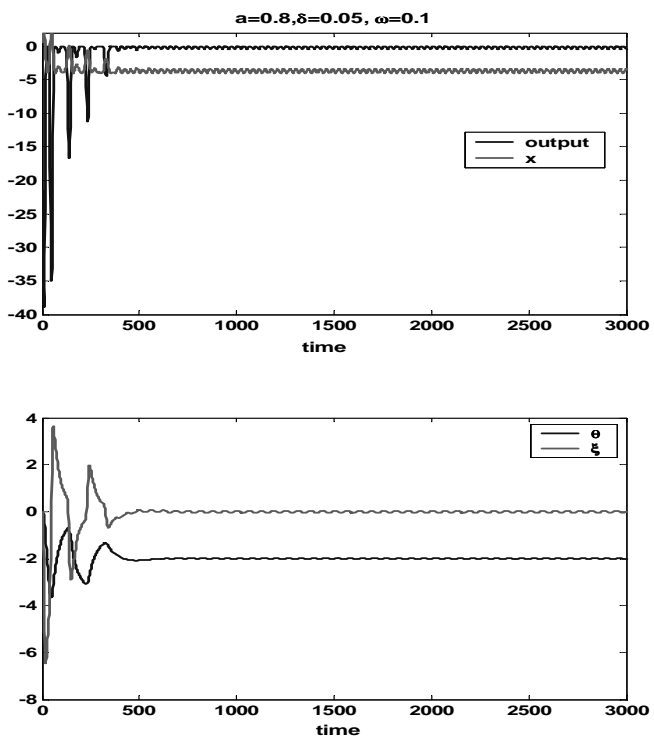

Fig. 2. The performance of the extremum seeking scheme

The initial condition is chosen as $\left[\begin{array}{lll}2 & 0 & 0\end{array}\right]^{T}$. The initial values are far away from the desired one $[-4.0,-2,0]^{T}$. We choose $\omega_{L}=4$ and $K=5$ in the simulations.

We hope that we can get a sufficiently large domain of the attraction, for example, $\Delta=200$ and $\nu=$ 0.1 , for the extremum seeking scheme. By choosing $a=0.8, \delta=0.05$ and $\omega=0.1$, we can see that the output converges to the vicinity of the extremum value. If we reduce $a$ such that $a=0.4$ while keeping $\delta=0.05$ and $\omega=0.1$, we can see from Figure 2 that $y(t)$ converges much closer to the optimal one $y^{*}=0$. However, it also can be observed that convergence speed of states slows down, especially, the convergence speed of $\theta$ and $\xi$, as shown in Figure 2. The simulation results illustrate that when we enlarge the domain of the attraction, we are slowing down the convergence speed.

\section{REFERENCES}

A. R. Teel, J. Peuteman and D. Aeyels (1999). Semi-global practical asymptotic stability and averaging. Systems \& Control Letters 37, 317323.

Christofides, P.D. and A. R. Teel (1996). Singular perturbation and input-to-state stability. IEEE Transactions on Automatic Control 41, 16451650.

Edwards, C.H. and D. Penney (1998). Calculus with Analytic Geometry Calculus of a Single Variable Multivariable Calculus. Prentice Hall. Upper Saddle River, New Jersey.

H.-H. Wang, S. Yeung and M. Krstić (2000). Experimental application of extremum seeking on an
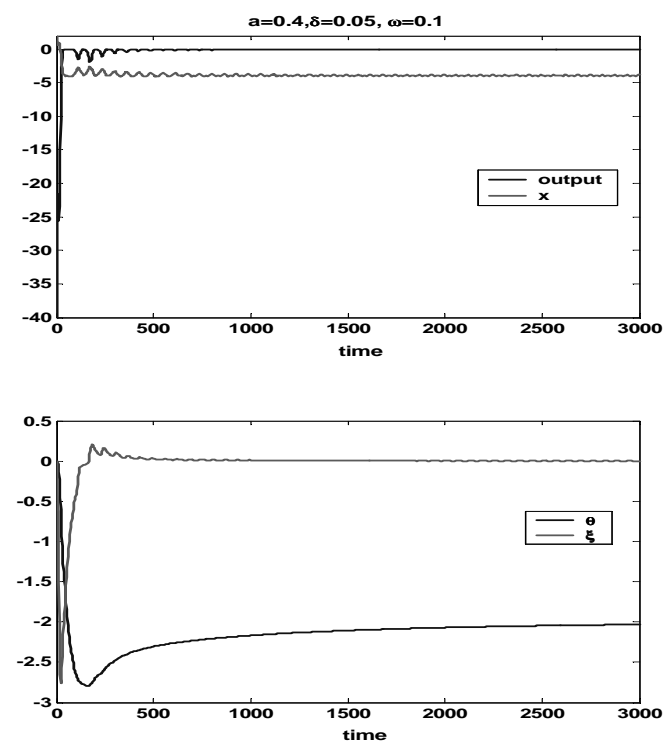

axial-flow compressor. IEEE Transactions on Control Systems Technology 8, 300-309.

Khalil, H. K. (2002). Nonlinear Systems. Prentice Hall. Upper Saddle River, New Jersey.

Krstić, M. (2000). Performance improvement and limitations in extremum seeking control. Systems and Control Letters 39, 313-326.

Krstić, M. and H. H. Wang (2000). Stability of extremum seeking feedback for general nonlinear dynamic systems. Automatica 36, 595-601.

M. Guay, D. Dochain, M. Perrier (2004). Adaptive extremum seekingcontrol of continuous stirred tank bioreactors with unknown growth kinetics. Automatica 40, 881-888.

Nešić, D. and A. R. Teel (2001). Input-to-state stability for nonlinear time-varying systems via averaging. Math. Contr. Sign. Sys. 14, 524533.

Peterson, K. S. and A. G. Stefanopoulou (2004). Extremum seeking control for soft landing of an electromechanical valve actuator. Automatica 40, 1063-1069.

Popović, D., M. Janković, S. Manger and A. R. Teel (2003). Extremum seeking methods for optimization of variable cam timing engine operation. In: Proc. American Control Conference. Denver, Colorado, USA. pp. 3136-3141.

Teel, A. R. (2000). Averaging with disturbances and closeness of solution. Systems \& Control Letters 40, 317-323.

Teel, A.R., L. Moreau and D. Nešić (2003). A unified framework for input-to-state stability in systems with two time scales. IEEE Transactions on Automatic Control 48, 1526-1544.

Wang, H.-H. and M. Krstić (2000). Extremum seeking for limit cycle minimization. IEEE Transactions on Automatic Control 45, 2432-2437. 